\title{
Study of Various Location Tracking Techniques for Centralized Location, Monitoring \& Control System
}

\author{
Priya Gupta ${ }^{1}$, S.S.Sutar ${ }^{2}$ \\ ${ }^{1}$ (M.E. Student Electronics \& Telecommunication, Rajiv Gandhi college of Engg., Mumbai University, India) \\ ${ }^{2}$ (Assistant Professor Electronics \& Telecommunication, Rajiv Gandhi college of Engg., Mumbai University, \\ India)
}

\begin{abstract}
Location tracking and control system require efficient resource management as well as reduction in their GSM network service cost. The data is transferred through GSM network by using SMS. This increases the system capability by efficiently managing tracking devices which could increase

in number as time goes by (GPS) has become a common functionality in handheld devices, and therefore, several location-tracking applications have been developed like GPS, GPRS, IMEI number, etc.,. Some techniques consume more time, less efficiency and more error rates. Location prediction is performed by using the current location, moving speed, and bearing of the target to predict its next location. When the distance between the predicted location and the actual location exceeds a certain threshold, the target transmits a short message to the tracker to update its current location. This paper presents a various tracking Techniques for Centralized location, Monitoring \& Control System.
\end{abstract}

Keywords: - Global Positioning System (GPS), Location Tracking, Mobile Phones, Prediction Algorithms, Short Message Service (SMS)..

\section{INTRODUCTION}

Real time tracking systems are usually suitable for large-scale systems which require real time data and consequently they require a certain amount of monthly budget to cover mobile internet cost using 3G/EDGE/GPRS. There are various cost-effective and efficient semi-offline systems developed and offered in the market. However, there are also many limitations and complications in the usage of the tracking devices; data transfer via SMS requires specific commands for integrated systems having a large number of devices. With the advent of GPS and the ubiquitous cellular network, real time tracking of object has become possible. Recent advancements in mobile technology allow Global Positioning System (GPS)-enabled mobile devices to provide a variety of real-time Location based Services (LBS). Among all those services, GPS navigation and location tracking are the most popular applications on mobile devices due to the usefulness and popularity during a trip. Formerly, a tourist has to look at paper maps or tour guide books during the trip, resulting in wasting time and easy to get lost. Without proper integrated device management, a system using SMS with a large number of tracking devices can ultimately become less efficient and effective

The present-day location tracking and control system can be classified according to the usage as follows:[6]

$\square$ Real Time Online

$\square$ Offline

$\square$ Semioffline

The Real Time Online device receives the real time position from the satellite and relays the information to the server at all time. The general online system is based on GPRS/EDGE/3G for the information relayed to the server [1]. This online tracking is commonly used with operations requiring locations at all time, e.g., logistics system, traffic system, taxi system, etc. The advantages lie in convenience of use. Managing and control is also efficient owing to the server that functions as a monitor and controller. However, 24-hour information relay results in high monthly expense from GPRS/EDGE/3G. Expense for the server as the system manager also incurs [2-4]. The Offline device also receives the present location from the satellite and records various data such as position, time, speed, etc. in its memory storage which is similar to the black box of an airplane. The information is not relayed to the user. The user has to check the recorded data when it is needed. Thus, there is no expense because the system is not connected to a network. The Semi-Offline receives the real time location from the satellite and sends it to the user as requested. Data sending can be based on GPRS/ $\mathrm{EDGE} / 3 \mathrm{G}$ or in the form of SMS. The advantage of the SMS semi-offline is alleviation of GPRS/EDGE/3G expense. The Semi-Offline is thus commonly used in the tracking system at present.[5] 


\subsection{Global Positioning System}

Using the Global Positioning System (GPS, a process used to establish a position at any point on the globe) the following two values can be determined anywhere on Earth. Speed and direction of travel (course) can be derived from this co-ordinates as well as the time. The co-ordinates and time values are determined by 28 satellites orbiting the Earth.

Recent advancements in mobile technology enable Global Positioning System (GPS)-enabled mobile devices to produce a range of period Location based Services (LBS). Among all those services, GPS navigation and placement pursuit are the foremost popular applications on mobile devices owing to the usefulness and recognition throughout a visit. Formerly, a tourist needs to scrutinize paper maps or guide books throughout the trip, leading to holdup and easy to urge lost. Therefore, electronic merchandise embedded with navigation functions became more and additional standard. By victimization GPS navigation capabilities of these electronic devices, the travelers will arrive to Associate in Nursing unknown destination speedily and effortlessly. At the in the meantime, a GPS tracking system is in a position to trace a selected target's movement and relay info involved wherever the target has traveled.

A GPS navigation device may be a device that may receive GPS signals for decisive the device's current location on Earth. It is very useful to develop a GPS navigation system on the Android platform with several Google resources. With Google Maps free navigation functionality, for software style, most navigation systems use GPS to get this position, and begin navigating when a user chooses destination or inputs its address. However, these steps don't seem to be terribly convenient to users.

\subsection{Location Tracking}

Location tracking refers to the attaining of the current position of object, stationary or moving. Localization may occur either via multilateration of radio signals between (several) radio towers of the network and the phone, or simply via GPS. To locate the Source using multilateration of radio signals, it must emit at least the roaming signal to contact the next nearby antenna tower, but the process does not require an active call. GSM is based on the signal strength to nearby antenna masts.

Mobile positioning, which includes location based service that discloses the actual coordinates of a mobile phone bearer, is a technology used by telecommunication companies to approximate the location of a mobile phone, and thereby also its user (bearer). The more properly applied term locating refers to the purpose rather than a positioning process.

\section{TRACKING DEVICES}

Tracking device is a device installed on a vehicle or an object. Its objective is to track and report current location data in latitude and longitude along with other important data. Furthermore, warning signal and alarm i.e. exceed speed, out-of-route information can also be sent from this kind of device. To complete this feature, tracking device also can follow commands for both pre-programmed and sent ones from the user i.e. electricity cutting-off, wire trapping or alarm on-off switching etc. The location tracking and device monitoring system in this research paper was developed focusing on using lowcost yet high-quality devices offered in the market. The system devices must be able to be attached to desired targets such as vehicles or luggages Lee et al. proposed a real-time location tracking system [1] for childcare or elderly care applications. It transmits the location information of the mobile device to a central GPS application server through the wireless networks. This application allows the server to simultaneously monitor multiple targets (e.g., elders or children). Lita et al. proposed an automobile localization system by using SMS [3]. And, Choi et al. assumed that the location information of a target is transmitted through wireless networks. Their work focused on proposing a geo location update scheme to decrease the update frequency [7]. Hameed et al. proposed a car monitoring and tracking system that uses both SMS and GPS to prevent car theft [5]. Anderson et al. proposed a transportation information system [6]. The proposed system, which is interconnected with the car alarm system, transmits alerts to the owner's mobile phone in the event of a car theft (e.g., activation of the car alarm, starting of the engine) or provides information for monitoring adolescent drivers (e.g., exceeding the speed limit or leaving a specific area). In this system, a hardware device called StarBox, which is equipped with a global system for mobile communications (GSM) modem and a GPS unit, is installed in a vehicle to track the vehicle's location. StarBox transmits short messages containing its GPS coordinates to the server at 30-s intervals. The users can send short messages to the server to determine the expected arrival time of buses at their locations. Although transmitting the Geo location information of a target via wireless networks is effective when both the target and the tracker are within Wi-Fi coverage area, the 802.11 wireless networks are not always accessible. When the target or the tracker is unable to access $\mathrm{Wi}-\mathrm{Fi}$, it is impossible to perform location tracking. Therefore, SMS is a relatively more reliable and flexible solution because of its widespread use (i.e., well-structured worldwide) [6], [8]. However, SMS is a user-pay service. In this paper, a novel method called location-based delivery (LBD), which combines SMS and GPS, is proposed, and further, a realistic system to perform precise location tracking 
is developed. The objective of this study is to minimize the transmission cost of a tracking system by minimizing the number of SMS transmissions while maintaining the location tracking accuracy. LBD mainly applies the following two proposed techniques: Location prediction and dynamic threshold. Location prediction is performed by using the current location, moving speed, and bearing of the target to predict its next location. When the distance between the predicted location and the actual location exceeds a certain threshold, the target transmits a short message to the tracker to update its current location. The dynamic threshold maintains the location tracking accuracy and number of short messages on the basis of the moving speed of the target.

GPS is widely used for target location because of its high positioning accuracy [1]-[3], [6], [8]. Related works have roughly classified the location tracking methods [3], [5], [6], [8], [9] using GPS and SMS as time based delivery and distance-based delivery. Additionally, GPS networks are well-constructed and widely accessible. Therefore, GPS networks are used, practically, for positioning an object by using triangulation. Time-based delivery is used to periodically transmit location update messages for tracking [3], [6], [10]. By contrast, distance-based delivery is used to transmit location update messages when the distance between the previously reported location and the current location exceeds a fixed-distance threshold [9]. However, SMS is a pay service. Therefore, the objective of this study is to minimize the number of SMS transmissions while simultaneously maintaining the location tracking accuracy. Time-based delivery is effective for tracking a target that is moving erratically. However, it exhibits a crucial flaw when the target remains stationary for an extended period, that is, it continues to periodically transmit many unnecessary short messages. The proposed approach differs from the distance-based delivery in two major aspects. First, LBD transmits a location update message when the distance between the predicted location and the actual location exceeds a certain threshold, rather than when the distance between two continuously reporting locations exceeds the threshold, a typical mechanism adopted by distance-based delivery. Thus, LBD can deliver fewer update messages. Additionally, LBD further reduces the number of required update messages while satisfactorily maintaining the location tracking accuracy because it adopts a dynamic threshold rather than the static threshold used in distance-based delivery. In our proposed work we applied multiple target detection and online map for efficient location determination. Existing methodologies focus on single target and offline processing, so the result prediction may take longer time duration. Those location tracking systems consumes more memory and GPS process consumes more battery power.

\section{TRACKING SYSTEM OVERVIEW}

The three key components designed in the system are as follows:
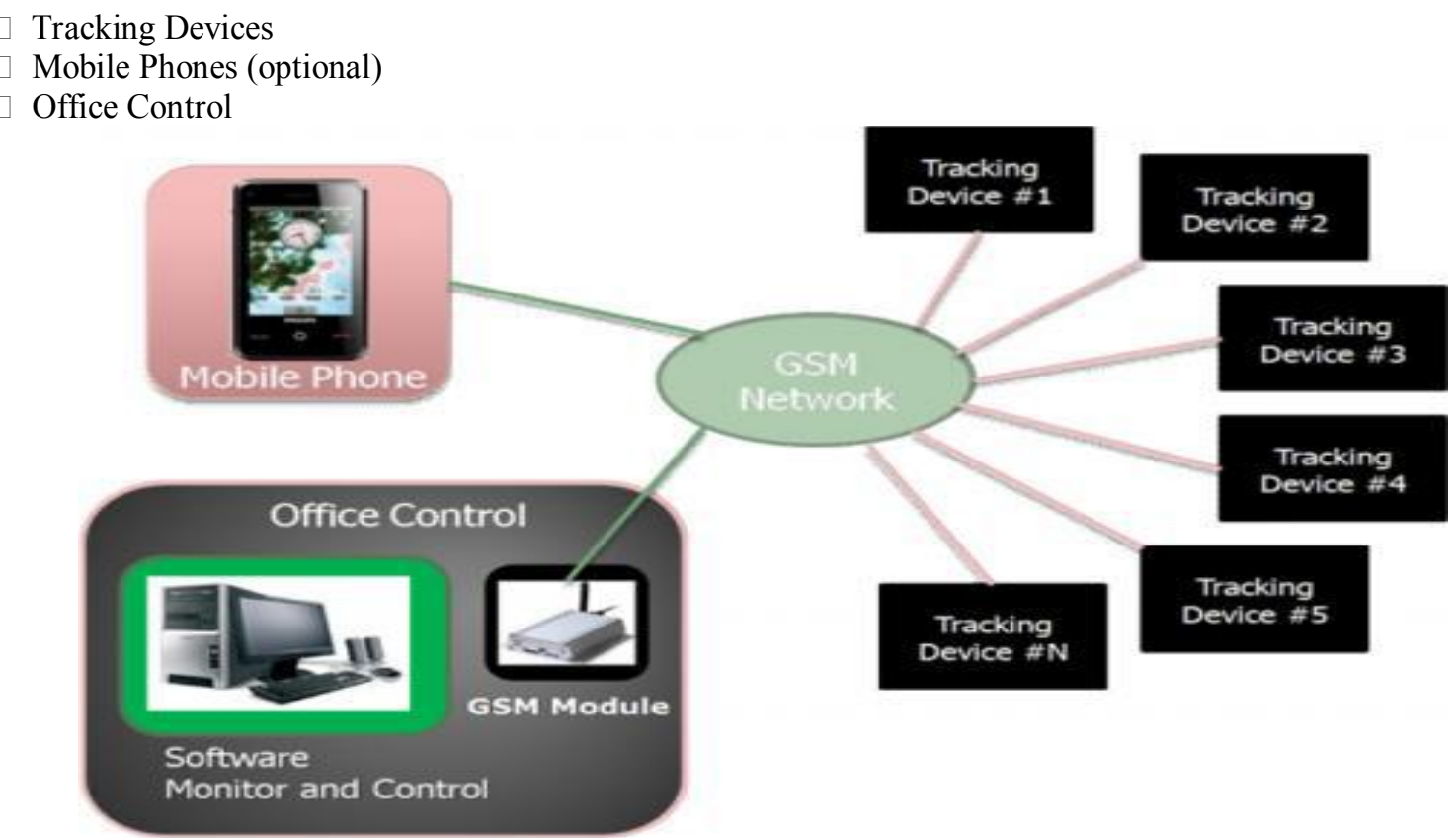

Figure 1. Location Tracking System Structure[6]

\subsection{TRACKING DEVICE}

The tracking device is an embedded system using MCU as a processing center, with GSM Module for wireless network and GPS Module for verifying coordination, location, and speed of the target. Tracking devices available in the market will be used because they are inexpensive, and have acceptable quality with 
wide selection. Since the devices can be applied in various purposes, a centralized system is required for device management.

\subsection{MOBILE PHONE}

The mobile phone is one of the options which gives users convenience in tracking and monitoring. Tracking is typically done by sending SMS to a tracking device. The responded data from the device is only its latitude and longitude which is difficult for interpretation. Therefore, the designed system uses Office Control (see Office Control) to convert latitude and longitude data and send URL to be opened on Google Map on a supported mobile phone. On a mobile phone which Google map is not supported, users can choose to retrieve the address information, instead of its coordination, for more practical information.

\subsection{OFFICE CONTROL}

The Office Control is a computer software (system server) controlling the overall system performance. It communicates with tracking devices attached to the target through GSM Network as well as transfer data and controls the devices.

\section{CONCLUSION}

A handful of studies have developed location tracking applications through SMS. However, SMS is a user-pay service. The number of SMS transmissions must be minimized while maintaining the location tracking accuracy within the acceptable range to reduce the transmission cost. Due to its low cost, it is very easy to integrate into other technologies like cell phone. the semi-offline location tracking, monitoring, and controlling system which can track and identify the target's location information on demand. It should also reduce cost in using GSM Network for small-scale logistics organizations by attaching tracking devices to the desired targets.

\section{REFERENCES}

[1] H. H. Lee, I. K. Park, and K. S. Hong, "Design and implementation of a mobile devices-based real-time location tracking," in Proc. UBICOMM, 2008, pp. 178-183.

[2] a Michael, Andrew McNamee, MG Michael, "The Emerging Ethics of Humancentric GPS Tracking and Monitoring”, Mobile Business, 2006. ICMB'06. International Conference on, pp. 1-10, 2006.

[3] I. Lita, I. B. Cioc, and D. A. Visan, "A new approach of automobile localization system using GPS and GSM/GPRS transmission," in Proc. ISSE, 2006, pp. 115-119.

[4] P. Perugu, "An innovative method using GPS tracking, WINS technologies for border security and tracking of vehicles," in Proc. RSTSCC, 2010, pp. 130-133.

[5] S. A. Hameed, O. Khalifa, M. Ershad, F. Zahudi, B. Sheyaa, and W. Asender, "Car monitoring, alerting, and tracking model: Enhancement with mobility and database facilities," in Proc. ICCCE, 2010, pp. 1-5.

[6] R. E. Anderson, A. Poon, C. Lustig, W. Brunette, G. Borriello, and B. E. Kolko, "Building a transportation information system using only GPS and basic SMS infrastructure," in Proc. ICTD, 2009, pp. 233-242.

[7] W. J. Choi and S. Tekinay, "Location-based services for next-generation wireless mobile networks," in Proc. IEEE VTC, 2003, pp. 1988-1992.

[8] R. E. Anderson, W. Brunette, E. Johnson, C. Lustig, A. Poon, C. Putnam, O. Salihbaeva, B. E. Kolko, and G. Borrielllo, "Experiences with a transportation information system that uses only GPS and SMS," in Proc. ICTD, 2010.

[9] A. Civilis, C. S. Jensen, and S. Pakalnis, "Techniques for efficient roadnetwork- based tracking of moving objects," IEEE Trans. Knowl. Data Eng., vol. 17, no. 5, pp. 698-712, 2005.

[10] M. Zahaby, P. Gaonjur, and S. Farajian, "Location tracking in GPS using Kalman filter through SMS," in Proc. IEEE EUROCON, 2009, pp. 1707- 1711.

[11] A. Civilis, C. S. Jensen, J. Nenortaite, and S. Pakalnis, "Efficient tracking of moving objects with precision guarantees," in Proc. MOBIQUITOUS, 2004, pp. 164-173.

[12] Y. Y. Xiao, H. Zhang, and H. Y. Wang, "Location prediction for tracking moving objects based on grey theory," in Proc. FSKD, 2007, pp. 390-394.

[13] P. H. Tseng, K. T. Feng, Y. C. Lin, and C. L. Chen, "Wireless location tracking algorithms for environments with insufficient signal sources," IEEE Trans. Mobile Comput., vol. 8, no. 12, pp. 16761689, 2009.

[14] R. Bajaj, S. L. Ranaweera, and D. P. Agrawal, "GPS: Location-tracking technology," Computer, vol. 35, no. 4, pp. 92-94, 2002.

[15] Movable Type Scripts. (2012 June). [Online]. Available: http://www.mova bletype.co.uk/scripts/latlong.html 